\title{
SKEW BRACES AND THE YANG-BAXTER EQUATION
}

\author{
L. GUARNIERI AND L. VENDRAMIN
}

\begin{abstract}
Braces were introduced by Rump to study non-degenerate involutive set-theoretic solutions of the Yang-Baxter equation. We generalize Rump's braces to the non-commutative setting and use this new structure to study not necessarily involutive non-degenerate set-theoretical solutions of the Yang-Baxter equation. Based on results of Bachiller and Catino and Rizzo, we develop an algorithm to enumerate and construct classical and non-classical braces of small size up to isomorphism. This algorithm is used to produce a database of braces of small size. The paper contains several open problems, questions and conjectures.
\end{abstract}

\section{INTRODUCTION}

The Yang-Baxter equation first appeared in theoretical physics and statistical mechanics in the works of Yang [42] and Baxter [4, 5] and it has led to several interesting applications in quantum groups and Hopf algebras, knot theory, tensor categories and integrable systems, see for example [27], [30] and [39]. In [14], Drinfeld posed the problem of studying this equation from the set-theoretical perspective.

Recall that a set-theoretical solution of the Yang-Baxter equation is a pair $(X, r)$, where $X$ is a set and

$$
r: X \times X \rightarrow X \times X, \quad r(x, y)=\left(\sigma_{x}(y), \tau_{y}(x)\right), \quad x, y \in X,
$$

is a bijective map such that

$$
(r \times \mathrm{id})(\mathrm{id} \times r)(r \times \mathrm{id})=(\mathrm{id} \times r)(r \times \mathrm{id})(\mathrm{id} \times r) .
$$

Such a map $r$ is usually called a braiding.

A solution $(X, r)$ is said to be non-degenerate if the maps $\sigma_{x}$ and $\tau_{x}$ are bijective for each $x \in X$, and $(X, r)$ is said to be involutive if $r^{2}=\mathrm{id}_{X \times X}$. The seminal works of Etingof, Schedler and Soloviev [15], and Gateva-Ivanova and Van den Bergh [24], discussed algebraic and geometrical interpretations and introduced several structures associated with the class of non-degenerate involutive solutions. Such solutions have been intensively studied, see for example [17, 18, 19], [21, 22, 23], [25, 26], $[20],[32,34],[6],[10],[11],[13],[28]$, and [40].

It was in studying involutive solutions that Rump introduced in [34] the brace structure. In [12], Cedó, Jespers and Okniński, defined a left brace as an abelian group $(A,+)$ with another group structure, defined via $(a, b) \mapsto a b$, such that the compatibility condition

$$
a(b+c)+a=a b+a c
$$

holds for all $a, b, c \in A$. This definition is equivalent to that of Rump.

This work is partially supported by CONICET, PICT-2014-1376, MATH-AmSud and ICTP. 
Many of the problems related to involutive solutions can be restated in terms of braces. Two prominent examples are the following:

- Is every finite solvable group an involutive Yang-Baxter group? Recall that an involutive Yang-Baxter group is a group isomorphic to the group generated by the set $\left\{\sigma_{x}: x \in X\right\}$, where

$$
r: X \times X \rightarrow X \times X, \quad r(x, y)=\left(\sigma_{x}(y), \tau_{y}(x)\right),
$$

is a non-degenerate involutive solution of the Yang-Baxter equation. Based on a sketch of proof of Rump [36], Bachiller [2] found a solvable finite group that is not an involutive Yang-Baxter group.

- Are there good methods to contruct all finite non-degenerate involutive solutions to the Yang-Baxter equation? Brute force seems not to be good enough. In [3], Bachiller, Cedó and Jespers, give a method to construct all finite solutions of a given size. For it to work, one needs the classification of left braces.

Non-involutive solutions were studied by Soloviev [38] and Lu, Yan and Zhu [29]. Such solutions have applications in knot theory, since they produce powerful knot and virtual knots invariants, see for example [31] and the references therein. The following question naturally arises: Is there an algebraic structure similar to the brace structure useful for studying non-involutive solutions? This paper introduces the notion of skew brace and provides an affirmative answer to the above question. Remarkably, this new structure provides the right algebraic framework to study involutive and non-involutive braidings and allows us to restate the main results of [29], [38] and [41].

As in the case of involutive solutions, the classification of finite skew braces is one of the main steps needed for constructing finite solutions of the Yang-Baxter equation. One of the main results of this paper is an explicit classification of classical and skew braces of small size. An algorithm to construct all non-isomorphic classical and skew braces of a given size is described. This heavily depends on results of Bachiller [2] and Catino and Rizzo [9]. This algorithm was used to build a database of classical and skew braces, a good source of examples that gives an explicit and direct way to approach some of the problems related to the Yang-Baxter equation. The database is available as a library for GAP [16] and Magma [8] immediately from the authors on request.

The paper is organized as follows. In Section 1 we extend braces to the noncommutative setting by defining skew braces, and state their main properties. We prove in Proposition 1.11 that skew braces are equivalent to bijective 1-cocycles. Section 2 is devoted to a study of quotients of skew braces. It is worth mentioning that the proofs in Section 1 and 2 are basically the same as for classical braces. In Section 3 the connection between skew braces and the Yang-Baxter equation is explored. In Theorem 3.1 we generalize a result of Rump and produce a canonical solution for each skew left brace. Some reconstruction theorems similar to those of Etingof, Schedler and Soloviev [15], Lu, Yan and Zhu [29] and Soloviev [38] are given at the end of this section. The method for constructing classical and skew braces is given in Section 4 . Section 5 discusses the algorithm that produces and enumerates classical and skew left braces and some consequences. Problems, questions and conjectures are discussed in Section 6. 


\section{SKEW LEFT BRACES}

Braces were introduced by Rump in [34] to study set-theoretical involutive solutions of the Yang-Baxter equation. The following definition generalizes braces to the non-commutative setting.

Definition 1.1. A skew left brace is a group $A$ (written multiplicatively) with an additional group structure given by $(a, b) \mapsto a \circ b$ such that

$$
a \circ(b c)=(a \circ b) a^{-1}(a \circ c)
$$

holds for all $a, b, c \in A$, where $a^{-1}$ denotes the inverse of a with respect to the group structure given by $(a, b) \mapsto a b$.

Of course Rump's left braces are examples of skew braces. These are braces where the group $(A, \cdot)$ is abelian.

Definition 1.2. $A$ homomorphism between two skew left braces $A$ and $B$ is a map $f: A \rightarrow B$ such that $f(a b)=f(a) f(b)$ and $f(a \circ b)=f(a) \circ f(b)$ for all $a, b \in A$. The kernel of $f$ is

$$
\text { ker } f=\{a \in A: f(a)=1\}
$$

where 1 denotes the identity of the group $(A, \cdot)$ with multiplication $a \cdot b=$ ab for all $a, b \in A$.

Example 1.3. Let $(A, \cdot)$ be a group. Then $A$ is a skew left brace with $a \circ b=a b$ for all $a, b \in A$. Similarly, $a \star b=b a$ defines a skew left brace structure over $A$. These braces are isomorphic if and only if $(A, \cdot)$ is abelian.

Example 1.4. Let $A$ and $B$ be groups and let $\alpha: A \rightarrow \operatorname{Aut}(B)$ be a group homomorphism. Then $A \times B$ has a skew left brace structure given by

$$
\begin{aligned}
& (a, b)\left(a^{\prime}, b^{\prime}\right)=\left(a a^{\prime}, b b^{\prime}\right), \\
& (a, b) \circ\left(a^{\prime}, b^{\prime}\right)=\left(a a^{\prime}, b \alpha_{a}\left(b^{\prime}\right)\right),
\end{aligned}
$$

where $a, a^{\prime} \in A$ and $b, b^{\prime} \in B$.

Example 1.5. Let $A$ and $B$ be groups and let $\alpha: A \rightarrow \operatorname{Aut}(B)$ be a group homomorphism. Assume that $A$ is abelian. Then $A \times B$ has a skew left brace structure given by

$$
\begin{aligned}
& (a, b)\left(a^{\prime}, b^{\prime}\right)=\left(a a^{\prime}, b \alpha_{a}\left(b^{\prime}\right)\right), \\
& (a, b) \circ\left(a^{\prime}, b^{\prime}\right)=\left(a a^{\prime}, b b^{\prime}\right),
\end{aligned}
$$

where $a, a^{\prime} \in A$ and $b, b^{\prime} \in B$.

Example 1.6. This example is motivated by the paper of Weinstein and Xu on the Yang-Baxter equation, see [41]. Let $A$ be a group and $A_{+}, A_{-}$be subgroups of $A$ such that $A$ admits a unique factorization as $A=A_{+} A_{-}$. Thus each $a \in A$ can be written in a unique way as $a=a_{+} a_{-}$for some $a_{+} \in A_{+}$and $a_{-} \in A_{-}$. The map

$$
A_{+} \times A_{-} \rightarrow A, \quad\left(a_{+}, a_{-}\right) \mapsto a_{+}\left(a_{-}\right)^{-1},
$$

is bijective. Using this map we transport the group structure of the direct product $A_{+} \times A_{-}$into the set $A$. For $a=a_{+} a_{-} \in A$ and $b=b_{+} b_{-} \in A$ let

$$
a \circ b=a_{+} b a_{-} .
$$

Then $(A, \circ)$ is a group. Furthermore, $A$ is a skew left brace. 
Lemma 1.7. Let $A$ be a skew left brace. Then the following properties hold:

(1) $1=1_{\circ}$, where 1 。 denotes the unit of the group $(A, \circ)$.

(2) $a \circ\left(b^{-1} c\right)=a(a \circ b)^{-1}(a \circ c)$ for all $a, b, c \in A$.

(3) $a \circ\left(b c^{-1}\right)=(a \circ b)(a \circ c)^{-1} a$ for all $a, b, c \in A$.

Proof. The first claim follows from (1.1) with $c=1_{\circ}$. To prove the second claim let $d=b c$. Then (1.1) becomes $a \circ d=(a \circ b) a^{-1}\left(a \circ b^{-1} d\right)$ and the claim follows. The third claim is proved similarly.

Remark 1.8. Let $A$ be a skew left brace. For each $a \in A$ the map

$$
\lambda_{a}: A \rightarrow A, \quad b \mapsto a^{-1}(a \circ b),
$$

is bijective with inverse $\lambda_{a}^{-1}: A \rightarrow A, b \mapsto \bar{a} \circ(a b)$, where $\bar{a}$ is the inverse of $a$ with respect to $\circ$. It follows that

$$
a \circ b=a \lambda_{a}(b), \quad a b=a \circ \lambda_{a}^{-1}(b)
$$

hold for all $a, b \in A$.

The following proposition extends results of Rump [34] and Gateva-Ivanova into the non-commutative setting, see [17, Proposition 3.3].

Proposition 1.9. Let $A$ be a set and assume that $A$ has two operations such that $(A, \cdot)$ and $(A, \circ)$ are groups. Assume that $\lambda: A \rightarrow \mathbb{S}_{A}, a \mapsto \lambda_{a}$, is given by $\lambda_{a}(b)=a^{-1}(a \circ b)$. The following are equivalent:

(1) $A$ is a skew left brace.

(2) $\lambda_{a \circ b}(c)=\lambda_{a} \lambda_{b}(c)$ for all $a, b, c \in A$.

(3) $\lambda_{a}(b c)=\lambda_{a}(b) \lambda_{a}(c)$ for all $a, b, c \in A$.

Proof. Let us first prove that $(1) \Longrightarrow(2)$. Let $a, b, c \in A$. Since $A$ is a brace and $a \circ b^{-1}=a(a \circ b)^{-1} a$ by Lemma 1.7,

$$
\begin{aligned}
\lambda_{a} \lambda_{b}(c) & =a^{-1}\left(a \circ \lambda_{b}(c)\right)=a^{-1}\left(a \circ\left(b^{-1}(b \circ c)\right)\right) \\
& =a^{-1}\left(a \circ b^{-1}\right) a^{-1}(a \circ b \circ c)=(a \circ b)^{-1}(a \circ b \circ c)=\lambda_{a \circ b}(c) .
\end{aligned}
$$

Now we prove $(2) \Longrightarrow(3)$. Since $a b=a \circ \lambda_{a}^{-1}(b)$ for all $a, b \in A$,

$$
\begin{aligned}
\lambda_{a}(b c) & =\lambda_{a}\left(b \circ \lambda_{b}^{-1}(c)\right)=a^{-1}\left(a \circ b \circ \lambda_{b}^{-1}(c)\right) \\
& =a^{-1}(a \circ b)(a \circ b)^{-1}\left(a \circ b \circ \lambda_{b}^{-1}(c)\right) \\
& =\lambda_{a}(b) \lambda_{a \circ b} \lambda_{b}^{-1}(c)=\lambda_{a}(b) \lambda_{a} \lambda_{b} \lambda_{b}^{-1}(c)=\lambda_{a}(b) \lambda_{a}(c) .
\end{aligned}
$$

Finally we prove that $(3) \Longrightarrow(1)$. Let $a, b, c \in A$. Then

$$
a^{-1}(a \circ(b c))=\lambda_{a}(b c)=\lambda_{a}(b) \lambda_{a}(c)=a^{-1}(a \circ b) a^{-1}(a \circ c),
$$

and hence $a \circ(b c)=(a \circ b) a^{-1}(a \circ c)$.

Corollary 1.10. Let $A$ be a skew left brace and

$$
\lambda:(A, \circ) \rightarrow \operatorname{Aut}(A, \cdot), \quad a \mapsto \lambda_{a}(b)=a^{-1}(a \circ b) .
$$

Then $\lambda$ is a group homomorphism.

Proof. It follows immediately from Proposition 1.9. 
Let $A$ and $G$ be groups and assume that $G \times A \rightarrow A,(g, a) \mapsto g \cdot a$, is a left action of $G$ on $A$ by automorphisms. A bijective 1-cocyle is a bijective map $\pi: G \rightarrow A$ such that

$$
\pi(g h)=\pi(g)(g \cdot \pi(h))
$$

for all $g, h \in G$.

Proposition 1.11. Over any group $(A, \cdot)$ the following data are equivalent:

(1) A group $G$ and a bijective 1-cocycle $\pi: G \rightarrow A$.

(2) A skew left brace structure over A.

Proof. Consider on $A$ a second group structure given by

$$
a \circ b=\pi\left(\pi^{-1}(a) \pi^{-1}(b)\right)
$$

for all $a, b \in A$. Since $\pi$ is a 1-cocycle and $G$ acts on $A$ by automorphisms,

$$
\begin{aligned}
a \circ(b c) & =\pi\left(\pi^{-1}(a) \pi^{-1}(b c)\right)=a\left(\pi^{-1}(a) \cdot(b c)\right) \\
& =a\left(\left(\pi^{-1}(a) \cdot b\right)\left(\pi^{-1}(a) \cdot c\right)\right)=(a \circ b) a^{-1}(a \circ c)
\end{aligned}
$$

holds for all $a, b, c \in A$.

Conversely, assume that $A$ is a skew left brace. Set $G=A$ with the multiplication $(a, b) \mapsto a \circ b$ and $\pi=$ id. By Corollary 1.10, $a \mapsto \lambda_{a}$, is a group homomorphism and hence $G$ acts on $A$ by automorphisms. Then (1.2) holds and therefore $\pi: G \rightarrow A$ is a bijective 1-cocycle.

Remark 1.12. The construction of Proposition 1.11 is categorical.

\section{IDEALS AND QUOTIENTS}

Definition 2.1. Let $A$ be a skew left brace. A normal subgroup $I$ of $(A, \circ)$ is said to be an ideal of $A$ if $I a=a I$ and $\lambda_{a}(I) \subseteq I$ for all $a \in A$.

Example 2.2. Let $f: A \rightarrow B$ be a skew brace homomorphism. Then $\operatorname{ker} f$ is an ideal of $A$ since

$$
f\left(\lambda_{a}(x)\right)=\lambda_{f(a)}(f(x))=1
$$

for all $x \in \operatorname{ker} f$ and $a \in A$.

Lemma 2.3. Let $A$ be a skew left brace and $I \subseteq A$ be an ideal. Then the following properties hold:

(1) $I$ is a normal subgroup of $(A, \cdot)$.

(2) $a \circ I=a I$ for all $a \in A$.

(3) $I$ and $A / I$ are skew braces.

Proof. Let $a, b \in I$. Then $a^{-1} b=\lambda_{a}(\bar{a} \circ b) \in I$ and hence $I$ is a subgroup of $(A, \cdot)$. Remark 1.8 implies

$$
a I=a \circ I=I \circ a=I a
$$

for all $a \in A$. Thus $I$ is a normal subgroup of $(A, \cdot)$ and hence it follows that $I$ is a skew left brace. Since the quotient groups $A / I$ for both operations are the same, $A / I$ is a skew left brace.

Definition 2.4. Let $A$ be a skew left brace. The socle of $A$ is

$$
\operatorname{Soc}(A)=\{a \in A: a \circ b=a b, b(b \circ a)=(b \circ a) b \text { for all } b \in A\} .
$$


Lemma 2.5. Let $A$ be a skew left brace. Then $\operatorname{Soc}(A)$ is an ideal of $A$ contained in the center of $(A, \cdot)$.

Proof. Let us first prove that $\operatorname{Soc}(A)$ is a subgroup of $(A, \circ)$. Clearly $1 \in \operatorname{Soc}(A)$. Let $a, a^{\prime} \in A$ and $b \in A$. Then $a \circ a^{\prime} \in \operatorname{Soc}(A)$ since

$$
\left(a \circ a^{\prime}\right) \circ b=a \circ\left(a^{\prime} \circ b\right)=a \circ\left(a^{\prime} b\right)=a\left(a^{\prime} b\right)=\left(a a^{\prime}\right) b=\left(a \circ a^{\prime}\right) b .
$$

Now since $\bar{a}=a^{-1} \in \operatorname{Soc}(A)$ and $b=\left(a a^{-1}\right) \circ b=a \circ\left(a^{-1} \circ b\right)=a\left(a^{-1} \circ b\right)$, it follows that $\bar{a} b=a^{-1} b=a^{-1} \circ b=\bar{a} \circ b$. Hence $\operatorname{Soc}(A)$ is a subgroup of $(A, \circ)$.

A direct calculation proves that

$$
\lambda_{b}(a)=b \circ a \circ \bar{b} \text { for all } a \in \operatorname{Soc}(A) \text { and } b \in A .
$$

Then it follows that $\operatorname{Soc}(A) \subseteq\left\{a \in A: a \circ b=a b, \lambda_{b}(a) \circ b=b \circ a\right.$ for all $\left.b \in A\right\}$.

Let $a \in \operatorname{Soc}(A)$ and $b, c \in A$. Then

$$
\begin{aligned}
& \lambda_{c} \lambda_{b}(a)=\lambda_{c \circ b}(a)=(c \circ b) \circ a \circ \overline{c \circ c}=c \circ \lambda_{b}(a) \circ \bar{c}, \\
& \lambda_{b}(a) c=b^{-1}(b \circ a) c=(b \circ a) b^{-1} c=b \circ(a(\bar{b} \circ c))=b \circ a \circ \bar{b} \circ c=\lambda_{b}(a) \circ c .
\end{aligned}
$$

Hence $\lambda_{b}(\operatorname{Soc}(A)) \subseteq \operatorname{Soc}(A)$ for all $b \in A$ and $\operatorname{Soc}(A)$ is a normal subgroup of $(A, \circ)$ by $(2.1)$.

Now we prove that $\operatorname{Soc}(A)$ is central in $(A, \cdot)$. Let $a \in \operatorname{Soc}(A), b \in A$ and $c=\bar{b}$. Since

$$
c \circ(b a)=(c \circ b) c^{-1}(c \circ a)=c^{-1}(c \circ a)=(c \circ a) c^{-1}=c \circ(a b),
$$

it follows that $b a=a b$.

\section{Braces And the Yang-BaXter Equation}

We turn our attention to the connection between skew left braces and settheoretic solutions of the Yang-Baxter equation. The following theorem generalizes a result of Rump to the non-commutative setting, see [12, Lemma 2].

Theorem 3.1. Let $A$ be a skew left brace. Then

$$
r_{A}: A \times A \rightarrow A \times A, \quad r_{A}(a, b)=\left(\lambda_{a}(b), \lambda_{\lambda_{a}(b)}^{-1}\left((a \circ b)^{-1} a(a \circ b)\right),\right.
$$

is a non-degenerate solution of the Yang-Baxter equation. Furthermore, $r_{A}$ is involutive if and only if $a b=b a$ for all $a, b \in A$.

Remark 3.2. Recall from [29] that a braiding operator over a group $(A, \circ)$ with multiplication $m:(a, b) \mapsto a \circ b$ is a bijective map $r: A \times A \rightarrow A \times A$ such that

(1) $r(a \circ b, c)=($ id $\times m) r_{12} r_{23}(a, b, c)$ for all $a, b, c \in A$,

(2) $r(a, b \circ c)=(m \times$ id $) r_{23} r_{12}(a, b, c)$ for all $a, b, c \in A$,

(3) $r(a, 1)=(1, a)$ and $r(1, a)=(a, 1)$ for all $a \in A$, and

(4) $m r(a, b)=a \circ b$ for all $a, b \in A$.

Braiding operators are equivalent to bijective 1-cocycles by [29, Theorem 2], and bijective 1-cocycles are equivalent to skew left braces by Proposition 1.11. One can prove that (3.1) is the braiding operator corresponding to the skew left brace $A$ under this equivalence.

Proof of Theorem 3.1. Every braiding operator is a non-degenerate solution of the Yang-Baxter equation by [29, Corollary 3]. Thus it is enough to prove that $r_{A}$ is a braiding operator on $(A, \circ)$. Set $r=r_{A}$. Since $\lambda_{a}^{-1}(b)=\bar{a} \circ(a b)$ for all $a, b \in A$,

$$
\lambda_{\lambda_{a}(b)}^{-1}\left((a \circ b)^{-1} a(a \circ b)\right)=\overline{\lambda_{a}(b)} \circ\left(\lambda_{a}(b)(a \circ b)^{-1} a(a \circ b)\right)=\overline{\lambda_{a}(b)} \circ(a \circ b)
$$


holds for all $a, b \in A$. Thus $\operatorname{mr}(a, b)=a \circ b$ for all $a, b \in A$. Clearly $r(a, 1)=(1, a)$ and $r(1, a)=(a, 1)$ for all $a \in A$. Let $a, b, c \in A$. By Corollary 1.10 one obtains

$$
\begin{aligned}
(\mathrm{id} \times m) r_{12} r_{23}(a, b, c) & =(\mathrm{id} \times m) r_{12}\left(a, \lambda_{b}(c), \overline{\lambda_{b}(c)} \circ b \circ c\right) \\
& =(\mathrm{id} \times m)\left(\lambda_{a} \lambda_{b}(c), \overline{\lambda_{a} \lambda_{b}(c)} \circ a \circ \lambda_{b}(c), \overline{\lambda_{b}(c)} \circ b \circ c\right) \\
& =\left(\lambda_{a} \lambda_{b}(c), \overline{\lambda_{a} \lambda_{b}(c)} \circ a \circ b \circ c\right)=r(a \circ b, c) .
\end{aligned}
$$

From Remark 1.8 and Proposition 1.9 one obtains that

$$
\lambda_{a}(b \circ c)=\lambda_{a}(b) \lambda_{a \circ b}(c)
$$

holds for all $a, b, c \in A$. From this formula one deduces that

$$
\lambda_{a}(b) \circ \lambda_{\overline{\lambda_{a}(b)} \circ a \circ b}(c)=\lambda_{a}(b) \circ \lambda_{\lambda_{a}(b)}^{-1} \lambda_{a} \lambda_{b}(c)=\lambda_{a}(b) \lambda_{a \circ b}(c)=\lambda_{a}(b \circ c) .
$$

holds for all $a, b, c \in A$. Then

$$
\begin{aligned}
& (m \times \mathrm{id}) r_{23} r_{12}(a, b, c)=(m \times \mathrm{id}) r_{23}\left(\lambda_{a}(b), \overline{\lambda_{a}(b)} \circ a \circ b, c\right) \\
& =(m \times \mathrm{id})\left(\lambda_{a}(b), \lambda_{\overline{\lambda_{a}(b)} \circ a \circ b}(c), \overline{\lambda_{\overline{\lambda_{a}(b)} \circ a \circ b}} \circ \overline{\lambda_{a}(b)} \circ a \circ b \circ c\right) \\
& =\left(\lambda_{a}(b) \circ \lambda_{\overline{\lambda_{a}(b) \circ a \circ b}}(c), \overline{\lambda_{\overline{\lambda_{a}(b)} \circ a \circ b}} \circ \overline{\lambda_{a}(b)} \circ a \circ b \circ c\right) \\
& =\left(\lambda_{a}(b \circ c), \overline{\lambda_{a}(b \circ c)} \circ a \circ b \circ c\right)=r(a, b \circ c) \text {. }
\end{aligned}
$$

for all $a, b, c \in A$.

Corollary 3.3. Let $A$ be a skew left brace and $X \subseteq A$ be a subset of $A$. Assume $b \lambda_{a}(x) b^{-1} \in X$ for all $x \in X$ and $a, b \in A$. Then $\left.r_{A}\right|_{X \times X}$ is a non-degenerate solution of the Yang-Baxter equation.

Proof. Clearly $\lambda_{a}(x) \in X$ and $b x b^{-1} \in X$ for all $a, b \in A$ and $x \in X$. Then it follows that $\lambda_{\lambda_{x}(y)}^{-1}\left((x \circ y)^{-1} x(x \circ y)\right) \in X$ for all $x, y \in X$. Now Theorem 3.1 implies the claim.

Example 3.4. Let $A$ and $B$ be groups and $\alpha: A \rightarrow \operatorname{Aut}(B)$ be a group homomorphism. The skew left brace of Example 1.4 yields the following solution:

$$
\begin{aligned}
& r:(A \times B) \times(A \times B) \rightarrow(A \times B) \times(A \times B), \\
& r\left(\left(a_{1}, b_{1}\right)\left(a_{2}, b_{2}\right)\right)=\left(\left(a_{2}, \alpha_{a_{1}}\left(b_{2}\right)\right),\left(a_{2}^{-1} a_{1} a_{2}, b_{2}^{-1} \alpha_{a_{2}^{-1}}\left(b_{1} \alpha_{a_{1}}\left(b_{2}\right)\right)\right)\right) .
\end{aligned}
$$

Example 3.5. Let $A$ be an abelian group, $B$ be a group and $\alpha: A \rightarrow \operatorname{Aut}(B)$ be a group homomorphism. The skew left brace of Example 1.5 yields the following solution:

$$
\begin{aligned}
& r:(A \times B) \times(A \times B) \rightarrow(A \times B) \times(A \times B), \\
& r\left(\left(a_{1}, b_{1}\right)\left(a_{2}, b_{2}\right)\right)=\left(\left(a_{2}, \alpha_{a_{1}^{-1}}\left(b_{2}\right)\right),\left(a_{1}, \alpha_{a_{1}^{-1}}\left(b_{2}^{-1}\right) b_{1} b_{2}\right)\right) .
\end{aligned}
$$

Example 3.6. Let $A$ be the skew left brace constructed in Example 1.6. The solution of Theorem 3.1 is similar to the solution constructed by Weinstein and Xu in terms of factorizable Poisson groups [41, Theorem 9.2]. The latter is $\tau r_{A} \tau$, where $r_{A}$ is the solution of Theorem 3.1 and $\tau(x, y)=(y, x)$ for all $x, y$.

Based on [29], for each skew left brace $A$ we relate the solution $r$ given by Theorem 3.1 to the so-called Venkov solution, i.e.

$$
s(a, b)=\left(b, b^{-1} a b\right), \quad a, b \in A .
$$


Proposition 3.7. Let $A$ be a skew left brace. For each $n \in \mathbb{N}$ the map $T_{n}$ given by

$$
T_{n}\left(a_{1}, \ldots, a_{n-1}, a_{n}\right)=\left(a_{1}, \lambda_{a_{1}}\left(a_{2}\right), \lambda_{a_{1} \circ a_{2}}\left(a_{3}\right), \ldots, \lambda_{a_{1} \circ \ldots \circ a_{n-1}}\left(a_{n}\right)\right)
$$

is invertible and satisfies

$$
T_{n} r_{i, i+1}=s_{i, i+1} T_{n}
$$

for all $n \geq 2$ and $i \in\{1, \ldots, n-1\}$, where $r_{i, i+1}$ and $s_{i, i+1}$ denote the actions of the braid group $\mathbb{B}_{n}$ on $A^{n}=A \times \cdots \times A$ (n-times) induced from $r$ and $s$ respectively.

Proof. A direct calculation shows that $T_{n}$ is invertible with inverse

$$
T_{n}^{-1}\left(a_{1}, \ldots, a_{n}\right)=\left(a_{1}, \lambda_{a_{1}}^{-1}\left(a_{2}\right), \lambda_{a_{1} a_{2}}^{-1}\left(a_{3}\right), \ldots, \lambda_{a_{1} \ldots a_{n-1}}^{-1}\left(a_{n}\right)\right) .
$$

To prove (3.2) we proceed by induction on $n$. The case $n=2$ follows from a direct calculation since

$$
\begin{aligned}
T_{2} r_{12}(a, b) & =T_{2}\left(\lambda_{a}(b), \lambda_{\lambda_{a}(b)}^{-1}\left((a \circ b)^{-1} a(a \circ b)\right)\right)=\left(\lambda_{a}(b),(a \circ b)^{-1} a(a \circ b)\right), \\
& =\left(\lambda_{a}(b), \lambda_{a}(b)^{-1} a \lambda_{a}(b)\right)=s_{12}\left(a, \lambda_{a}(b)\right)=s_{12} T_{2}(a, b)
\end{aligned}
$$

holds for all $a, b \in A$. So assume that the claim holds for $n-1$. Since $T_{n} r_{1,2}=s_{1,2} T_{n}$ is the same as $T_{2} r=s T_{2}$, we need to prove (3.2) for all $i \in\{2, \ldots, n-1\}$. Write

$$
T_{n}=U_{n}\left(\mathrm{id} \times T_{n-1}\right),
$$

where

$$
U_{n}\left(a_{1}, \ldots, a_{n-1}, a_{n}\right)=\left(a_{1}, \lambda_{a_{1}}\left(a_{2}\right), \ldots, \lambda_{a_{1}}\left(a_{n-1}\right), \lambda_{a_{1}}\left(a_{n}\right)\right) .
$$

Since each $\lambda_{a}$ is an automorphism of $(A, \cdot)$, it follows that $U_{n} s_{i, i+1}=s_{i, i+1} U_{n}$ for $i \geq 2$ and hence (3.2) holds for all $i \geq 2$.

Remark 3.8. Proposition 3.7 also follows from [28, Proposition 6.2]. The map $T_{n}$ is the so-called guitar map, see for example $[28, \S 6]$.

The universal construction of Lu, Yan and Zhu, given in [29, Theorem 9] can be restated in the language of skew left braces. This was done by Rump in the case of involutive solutions, see [34]. Recall that the enveloping (or structure) group of a solution $(X, r)$ is the group $G(X, r)$ generated by the elements of $X$ with relations

$$
x \circ y=\sigma_{x}(y) \circ \tau_{y}(x), \quad x, y \in X .
$$

Let $\iota: X \rightarrow G(X, r)$ be the canonical map.

Theorem 3.9. Let $X$ be a set, $r: X \times X \rightarrow X \times X, r(x, y)=\left(\sigma_{x}(y), \tau_{y}(x)\right)$ be a non-degenerate solution of the Yang-Baxter equation. Then there exists a unique skew left brace structure over $G(X, r)$ such that its associated solution $r_{G}$ satisfies

$$
r_{G}(\iota \times \iota)=(\iota \times \iota) r .
$$

Furthermore, if $B$ is a skew left brace and $f: X \rightarrow B$ is a map such that $(f \times f) r=$ $r_{B}(f \times f)$, then there exists a unique group homomorphism $\phi: G(X, r) \rightarrow B$ such that $f=\phi \iota$ and $(\phi \times \phi) r_{G}=r_{B}(\phi \times \phi)$.

Proof. The claim follows from the universal construction of [29, Theorem 9] and the equivalence between braiding operators and skew braces, see Remark 3.2.

The following corollary is essentially [38, Theorem 2.6].

Corollary 3.10. Let $(X, r)$ be a finite non-degenerate solution of the Yang-Baxter equation. Then $G(X, r) / \operatorname{Soc}(G(X, r))$ is a finite skew left brace. 
Proof. It follows from Theorem 3.9 and Lemma 2.5.

\section{Constructing skew Braces}

Let $A$ be a group. The holomorph of $A$ is the group $\operatorname{Hol}(A)=\operatorname{Aut}(A) \ltimes A$, where the product is given by

$$
(f, a)(g, b)=(f g, a f(b))
$$

for all $a, b \in A$ and $f, g \in \operatorname{Aut}(A)$. Any subgroup $H$ of $\operatorname{Hol}(A)$ acts on $A$

$$
(f, x) \cdot a=\pi_{2}((f, x)(\mathrm{id}, a))=x f(a), \quad a, x \in A, f \in \operatorname{Aut}(A),
$$

where $\pi_{2}: \operatorname{Hol}(A) \rightarrow A,(f, a) \mapsto a$. In particular $\operatorname{Hol}(A)$ acts transitively on $A$ and the stabilizer of any $a \in A$ is isomorphic to $\operatorname{Aut}(A)$.

Recall that a subgroup $H$ of $\operatorname{Hol}(A)$ is regular if for each $a \in A$ there exists a unique $(f, x) \in H$ such that $x f(a)=1$. The following result is well-known.

Lemma 4.1. Let $A$ be a group and $H$ be a regular subgroup of $\operatorname{Hol}(A)$. Then $\left.\pi_{2}\right|_{H}: H \rightarrow A,(f, a) \mapsto a$, is bijective.

Proof. We first prove that $\left.\pi_{2}\right|_{H}$ is injective. Let $(f, a),(g, b) \in H$ be such that $\pi_{2}(f, a)=\pi_{2}(g, b)$. Then $a=b$. Since $H$ is a subgroup,

$$
(f, a)^{-1}=\left(f^{-1}, f^{-1}\left(a^{-1}\right)\right) \in H, \quad(g, a)^{-1}=\left(g^{-1}, g^{-1}\left(a^{-1}\right)\right) \in H,
$$

and hence $f=g$ since $f^{-1}(a) f^{-1}\left(a^{-1}\right)=g^{-1}(a) g^{-1}\left(a^{-1}\right)=1$ and $H$ is a regular subgroup.

Now we prove that $\left.\pi_{2}\right|_{H}$ is surjective. Let $a \in A$. The regularlity of $H$ implies the existence of an automorphism $f \in \operatorname{Aut}(A)$ such that $\left(f, f\left(a^{-1}\right)\right) \in H$. Then $\left(f^{-1}, a\right) \in H$ and the claim follows.

The following theorem goes back to Bachiller [2]. The proof in our case is the same as for braces. It is a generalization of a result of Catino and Rizzo [9].

Theorem 4.2. Let $A$ be skew left brace. Then $\left\{\left(\lambda_{a}, a\right): a \in A\right\}$ is a regular subgroup of $\operatorname{Hol}(A, \cdot)$. Conversely, if $(A, \cdot)$ is a group and $H$ is a regular subgroup of $\operatorname{Hol}(A, \cdot)$, then $A$ is a skew left brace with $(A, \circ) \simeq H$, where

$$
a \circ b=a f(b)
$$

and $\left(\left.\pi_{2}\right|_{H}\right)^{-1}(a)=(f, a) \in H$.

Proof. Since $\lambda$ is a group homomorphism and $a \lambda_{a}(b)=a \circ b$ for all $a, b \in A$, it follows that $\left\{\left(\lambda_{a}, a\right): a \in A\right\}$ is a subgroup of $\operatorname{Hol}(A, \cdot)$. Since $(A, \circ)$ is a group, the regularlity also follows.

Assume now that $H$ is a regular subgroup. By Lemma 4.1, $\left.\pi_{2}\right|_{H}$ is bijective. Use the bijection $\left.\pi_{2}\right|_{H}$ to transport the product of $H$ into $A$ :

$$
a \circ b=\left.\pi_{2}\right|_{H}\left(\left(\left.\pi_{2}\right|_{H}\right)^{-1}(a)\left(\left.\pi_{2}\right|_{H}\right)^{-1}(b)\right)=a f(b),
$$

where $a, b \in A$ and $\left(\left.\pi_{2}\right|_{H}\right)^{-1}(a)=(f, a) \in H$. Then $(A, \circ)$ is a group and $A$ is a skew left brace since

$$
a \circ(b c)=a f(b c)=a f(b) f(c)=a f(b) a^{-1} a f(c)=(a \circ b) a^{-1}(a \circ c)
$$

holds for all $a, b, c \in A$. 
Proposition 4.3. Let $A$ be a group. There exists a bijective correspondence between skew left brace structures over $A$ and regular subgroups of $\operatorname{Hol}(A)$. Moreover, isomorphic skew braces structures over A correspond to conjugate subgroups of $\mathrm{Hol}(A)$ by elements of $\operatorname{Aut}(A)$.

Proof. Assume that the group $A$ has two skew left brace structures given by $(a, b) \mapsto$ $a \circ b$ and $(a, b) \mapsto a \times b$ and that $\phi \in \operatorname{Aut}(A, \cdot)$ satisfies $\phi(a \circ b)=\phi(a) \times \phi(b)$ for all $a, b \in A$. We claim that $\left\{\left(\lambda_{a}, a\right): a \in A\right\}$ and $\left\{\left(\mu_{a}, a\right): a \in A\right\}$, where $\lambda_{a}(b)=a^{-1}(a \circ b)$ and $\mu_{a}(b)=a^{-1}(a \times b)$, are conjugate by $\phi$. Since

$$
\phi \lambda_{a} \phi^{-1}(b)=\phi\left(a^{-1}\left(a \circ \phi^{-1}(b)\right)=\phi(a)^{-1}(\phi(a) \times b)=\mu_{\phi(a)}(b),\right.
$$

one obtains that $\phi\left(\lambda_{a}, a\right) \phi^{-1}=\left(\mu_{\phi(a)}, \phi(a)\right)$ and hence the claim follows.

Conversely, let $H$ and $K$ be regular subgroups of $\operatorname{Hol}(A)$ and asssume that there exists $\phi \in \operatorname{Aut}(A, \cdot)$ such that $\phi^{-1} H \phi=K$. Let $(f, a)=\left(\left.\pi_{2}\right|_{H}\right)^{-1}(a) \in H$, $(g, a)=\left(\left.\pi_{2}\right|_{K}\right)^{-1}(a) \in K$ and write $a \circ b=a f(b)$ and $a \times b=a g(b)$. Since $\phi(f, a) \phi^{-1}=\left(\phi f \phi^{-1}, \phi(a)\right) \in K$, it follows that $\left(\left.\pi_{2}\right|_{K}\right)^{-1}(\phi(a))=\left(\phi f \phi^{-1}, \phi(a)\right)$. Then, since $\phi \in \operatorname{Aut}(A, \cdot)$,

$$
\phi(a) \times \phi(b)=\phi(a)\left(\phi f \phi^{-1}\right)(\phi(b))=\phi(a) \phi(f(b))=\phi(a f(b))=\phi(a \circ b)
$$

and hence the skew left braces corresponding to $H$ and $K$ are isomorphic.

\section{Computational Results}

We first present the algorithm used to enumerate skew left brace structures over a given group $A$. The algorithm uses Theorem 4.2.

Algorithm 5.1. Let $A$ be a finite group. To construct all skew left brace structures over $A$ we proceed as follows:

(1) Compute the holomorph $\operatorname{Hol}(A)$ of $A$.

(2) Compute the list of regular subgroups of $\operatorname{Hol}(A)$ of order $|A|$ up to conjugation by elements of $\operatorname{Aut}(A)$.

(3) For each representative $H$ of regular subgroups of $\mathrm{Hol}(A)$ construct the map $p: A \rightarrow H$ given by $a \mapsto\left(f, f(a)^{-1}\right)$, where $\left(f, f(a)^{-1}\right) \in H$. The triple $(H, A, p: A \rightarrow H)$ yields a skew left brace structure over $A$ with multiplication given by $a \circ b=p^{-1}(p(a) p(b))$ for all $a, b \in A$.

Remark 5.2. To enumerate all skew left brace structures over $A$ the third step of Algorithm 5.1 is not needed.

Remark 5.3. Recall that a left brace is an abelian group $(A,+)$ with another group structure, defined via the multiplication $(a, b) \mapsto a b$ such that $a(b+c)+a=a b+a c$ holds for all $a, b, c \in A$. Left braces with additive group isomorphic to a given group $A$ can be constructed applying Algorithm 5.1 to the abelian group $A$. In the second step of Algorithm 5.1 it is enough to compute the list of regular solvable subgroups of $\operatorname{Hol}(A)$, since the multiplicative group of a left brace is solvable by $[15$, Proposition 2.5].

Algorithm 5.1 was implemented both in GAP and Magma with different performances and were run on a Intel(R) Core(TM) i5-4440 CPU @ $3.10 \mathrm{GHz}$ with 16gb of RAM, under Linux. 
5.1. Skew left braces. For $n \in \mathbb{N}$ let $c(n)$ be the number of non-isomorphic skew left braces of size $n$.

The number of skew left braces of size $n \leq 30$ has been determined using Algorithm 5.1. Table 5.1 shows some values of $c(n)$. The calculation took about twenty minutes.

TABLE 5.1. The number of non-isomorphic skew left braces.

\begin{tabular}{|c|ccccccccccccccc|}
\hline$n$ & 1 & 2 & 3 & 4 & 5 & 6 & 7 & 8 & 9 & 10 & 11 & 12 & 13 & 14 & 15 \\
$c(n)$ & 1 & 1 & 1 & 4 & 1 & 6 & 1 & 47 & 4 & 6 & 1 & 38 & 1 & 6 & 1 \\
\hline$n$ & 16 & 17 & 18 & 19 & 20 & 21 & 22 & 23 & 24 & 25 & 26 & 27 & 28 & 29 & 30 \\
$c(n)$ & 1605 & 1 & 49 & 1 & 43 & 8 & 6 & 1 & 855 & 4 & 6 & 101 & 29 & 1 & 36 \\
\hline
\end{tabular}

5.2. Left braces. For $n \in \mathbb{N}$ let $b(n)$ be the number of non-isomorphic left braces of size $n$.

The number of left braces (up to isomorphism) of size $n \leq 120$ has been determined using Algorithm 5.1. Table 5.3 shows some values of $b(n)$ and Table 5.2 gives runtimes for our Magma implementation for some examples. The construction of left braces requires considerably more CPU time, see Table 5.4 for some examples.

TABLE 5.2. Some runtimes for enumerating left braces of size $n$.

\begin{tabular}{|r|r|r|}
\hline$n$ & CPU time & $b(n)$ \\
\hline 16 & 1 hour & 357 \\
48 & 18 hours & 1708 \\
54 & 5 minutes & 80 \\
72 & 1 hour & 489 \\
80 & 17 hours & 1985 \\
100 & 15 secs & 51 \\
108 & 28 hours & 494 \\
112 & 12 hours & 1671 \\
\hline
\end{tabular}

With current computational resources, we were not able to compute the number of non-isomorphic left braces of orders 32, 64, 81 and 96 .

5.3. Two-sided left braces (radical rings). Recall that a brace $B$ is a two-sided brace if $(a+b) c+c=a c+b c$ holds for all $a, b, c \in B$. Two-sided braces are in bijective correspondence with radical rings [33]. Recall that a non-zero radical ring is a ring $R$ without identity such that for each $x \in R$ there is $y \in R$ such that $x+y+x y=0$. Assume that $R$ is a radical ring. Then the circle operation,

$$
a \circ b=a b+a+b, \quad a, b \in R,
$$

makes $(R,+, \circ)$ into a two-sided brace. Conversely, if $A$ is a two-sided brace, the operation $a * b=a b-a-b, a, b \in A$ makes $(A,+, *)$, into a radical ring.

To test whether a left brace is a two-sided brace one has the following lemma of Gateva-Ivanova, see [17, Corollary 3.5].

Lemma 5.4 (Gateva-Ivanova). Let $A$ be a left brace. Then $A$ is a two-sided brace if and only if

$$
b c \lambda_{a b c}^{-1}(c)=c \lambda_{a c}^{-1}\left(\lambda_{a}(b) c\right)
$$


TABLE 5.3. The number of non-isomorphic left braces.

\begin{tabular}{|c|cccccccccccc|}
\hline$n$ & 1 & 2 & 3 & 4 & 5 & 6 & 7 & 8 & 9 & 10 & 11 & 12 \\
$b(n)$ & 1 & 1 & 1 & 4 & 1 & 2 & 1 & 27 & 4 & 2 & 1 & 10 \\
\hline$n$ & 13 & 14 & 15 & 16 & 17 & 18 & 19 & 20 & 21 & 22 & 23 & 24 \\
$b(n)$ & 1 & 2 & 1 & 357 & 1 & 8 & 1 & 11 & 2 & 2 & 1 & 96 \\
\hline$n$ & 25 & 26 & 27 & 28 & 29 & 30 & 31 & 32 & 33 & 34 & 35 & 36 \\
$b(n)$ & 4 & 2 & 37 & 9 & 1 & 4 & 1 & $?$ & 1 & 2 & 1 & 46 \\
\hline$n$ & 37 & 38 & 39 & 40 & 41 & 42 & 43 & 44 & 45 & 46 & 47 & 48 \\
$b(n)$ & 1 & 2 & 2 & 106 & 1 & 6 & 1 & 9 & 4 & 2 & 1 & 1708 \\
\hline$n$ & 49 & 50 & 51 & 52 & 53 & 54 & 55 & 56 & 57 & 58 & 59 & 60 \\
$b(n)$ & 4 & 8 & 1 & 11 & 1 & 80 & 2 & 91 & 2 & 2 & 1 & 28 \\
\hline$n$ & 61 & 62 & 63 & 64 & 65 & 66 & 67 & 68 & 69 & 70 & 71 & 72 \\
$b(n)$ & 1 & 2 & 11 & $?$ & 1 & 4 & 1 & 11 & 1 & 4 & 1 & 489 \\
\hline$n$ & 73 & 74 & 75 & 76 & 77 & 78 & 79 & 80 & 81 & 82 & 83 & 84 \\
$b(n)$ & 1 & 2 & 5 & 9 & 1 & 6 & 1 & 1985 & $?$ & 2 & 1 & 34 \\
\hline$n$ & 85 & 86 & 87 & 88 & 89 & 90 & 91 & 92 & 93 & 94 & 95 & 96 \\
$b(n)$ & 1 & 2 & 1 & 90 & 1 & 16 & 1 & 9 & 2 & 2 & 1 & $?$ \\
\hline$n$ & 97 & 98 & 99 & 100 & 101 & 102 & 103 & 104 & 105 & 106 & 107 & 108 \\
$b(n)$ & 1 & 8 & 4 & 51 & 1 & 4 & 1 & 106 & 2 & 2 & 1 & 494 \\
\hline$n$ & 109 & 110 & 111 & 112 & 113 & 114 & 115 & 116 & 117 & 118 & 119 & 120 \\
$b(n)$ & 1 & 6 & 2 & 1671 & 1 & 6 & 1 & 11 & 11 & 2 & 1 & 395 \\
\hline
\end{tabular}

TABLE 5.4. Some runtimes for constructing left braces of size $n$.

\begin{tabular}{|r|r|r|}
\hline$n$ & CPU time & $b(n)$ \\
\hline 16 & 3 hours & 357 \\
54 & 40 minutes & 80 \\
72 & 24 hours & 489 \\
112 & 5 days & 1671 \\
\hline
\end{tabular}

for all $a, b, c \in A$.

For $n \in \mathbb{N}$ let $t(n)$ be the number of non-isomorphic two-sided braces of size $n$. Using the database of left braces constructed with Algorithm 5.1 and Lemma 5.4 one computes $t(n)$. Table 5.5 shows the value of $t(n)$ for $n \leq 24$.

TABLE 5.5. The number of non-isomorphic two-sided braces.

\begin{tabular}{|c|cccccccccccc|}
\hline$n$ & 1 & 2 & 3 & 4 & 5 & 6 & 7 & 8 & 9 & 10 & 11 & 12 \\
$t(n)$ & 1 & 1 & 1 & 4 & 1 & 1 & 1 & 22 & 4 & 1 & 1 & 4 \\
\hline$n$ & 13 & 14 & 15 & 16 & 17 & 18 & 19 & 20 & 21 & 22 & 23 & 24 \\
$t(n)$ & 1 & 1 & 1 & 221 & 1 & 4 & 1 & 4 & 1 & 1 & 1 & 22 \\
\hline
\end{tabular}

Remark 5.5. For information on square-free two-sided braces, see [12]. These braces are defined by nilpotent groups of class $\leq 2$. 


\section{Further Questions}

In this section we collect some questions and conjectures that appear naturally after inspecting Table 5.3.

6.1. Left braces. We first collect some problems and conjectures related to the number of left braces.

Problem 6.1. Compute b(32), b(64), b(81) and $b(96)$.

Table 5.3 suggests the following conjectures.

Conjecture 6.2. Let $p>3$ be a prime number. Then

$$
b(4 p)= \begin{cases}11 & \text { if } m \equiv 1 \bmod 4, \\ 9 & \text { if } m \equiv 3 \bmod 4 .\end{cases}
$$

Conjecture 6.3. Let $p>3$ be a prime. Then

$$
b(9 p)= \begin{cases}14 & \text { if } p \equiv 1 \bmod 9 \\ 4 & \text { if } p \equiv 2,5 \bmod 9 \\ 11 & \text { if } p \equiv 4,7 \bmod 9 .\end{cases}
$$

Conjecture 6.4. Let $p, q$ be prime numbers such that $p<q$ and $q \not \equiv 1 \bmod p$. Then $b\left(p^{2} q\right)=4$.

We have used [7] and computer calculations to show that Conjectures 6.2 and 6.3 are true up to $p=997$. In [37], Agata Smoktunowicz proved that Conjecture 6.4 is true.

6.2. Quaternionic braces. We now consider an important family of braces. Recall that for $m \in \mathbb{N}$ the generalized quaternion group is the group

$$
Q_{4 m}=\left\langle a, b: a^{m}=b^{2}, a^{2 m}=1, b^{-1} a b=a^{-1}\right\rangle .
$$

Definition 6.5. A brace is a quaternion brace if its multiplicative group is isomorphic to some quaternion group.

Conjecture 6.6. For $m \in \mathbb{N}$ let $q(4 m)$ be the number of isomorphism classes of quaternion braces of size $4 m$. Then for $m>2$

$$
q(4 m)= \begin{cases}2 & \text { if } m \text { is odd } \\ 7 & \text { if } m \equiv 0 \bmod 8 \\ 9 & \text { if } m \equiv 4 \bmod 8 \\ 6 & \text { if } m \equiv 2 \bmod 8 \text { or } m \equiv 6 \bmod 8 .\end{cases}
$$

We have checked Conjecture 6.6 for all $m \leq 512$. It seems natural to ask the following questions.

Question 6.7. Which finite abelian groups appear as the additive group of a quaternion brace?

For $m \in\{2, \ldots, 512\}$ the additive group of a quaternion brace of size $m$ is isomorphic to one of the following groups:

$$
\mathbb{Z}_{4 m}, \mathbb{Z}_{2 m} \times \mathbb{Z}_{2}, \mathbb{Z}_{m} \times \mathbb{Z}_{2} \times \mathbb{Z}_{2}, \mathbb{Z}_{m} \times \mathbb{Z}_{4}, \mathbb{Z}_{m / 2} \times \mathbb{Z}_{2} \times \mathbb{Z}_{2} \times \mathbb{Z}_{2}
$$

By inspection, one sees that the groups $\mathbb{Z}_{m} \times \mathbb{Z}_{2}^{2}$ appear whenever $m \equiv 2,4,6 \bmod 8$ and the groups $\mathbb{Z}_{m} \times \mathbb{Z}_{4}$ and $\mathbb{Z}_{m / 2} \times \mathbb{Z}_{2}^{3}$ appear whenever $m \equiv 4 \bmod 8$. 
Question 6.8. For $m>2$ let $A$ be a finite abelian group size $4 m$. Compute the number of isomorphism classes of quaternion braces of size $4 m$ with additive group isomorphic to $A$.

In $[3, \S 5]$ quaternion braces of size $2^{k}$ are mentioned as an important class of braces which could be useful to classify a certain family of involutive non-degenerate solutions of the Yang-Baxter equation. Conjecture 6.6 implies the following:

Conjecture 6.9. There are seven classes of isomorphism of quaternion braces of size $2^{k}$ for $k>4$.

Conjecture 6.9 was verified for all $k \in\{5,6,7,8,9\}$. Table 6.1 sums up our findings related to this important subclass of braces.

TABle 6.1. Number of braces with multiplicative group isomorphic to the quaternion group $Q_{2^{k}}$ with $k>4$.

\begin{tabular}{|c|c|}
\hline Additive Group & Number of Braces \\
\hline $\mathbb{Z}_{2^{k}}$ & 1 \\
\hline $\mathbb{Z}_{2^{k-1}} \times \mathbb{Z}_{2}$ & 6 \\
\hline
\end{tabular}

Remark 6.10. The classification of left braces over cyclic groups was done by Rump in [35]. He proved that if a left brace $A$ has additive group isomorphic to $\mathbb{Z} / p^{k}$, where $p>2$ is a prime number, then $(A, \cdot) \simeq \mathbb{Z} / p^{k}$. According to [3], the converse holds for all $p$. In [1], Bachiller classified left braces of size $p^{2}$ and $p^{3}$, where $p$ is a prime number. The techniques used in these papers might prove useful to address the questions, problems and conjectures in this section.

\section{ACKNOWLEDGEMENTS}

We thank Leandro del Pezzo for the computer where some calculations were performed, David Bachiller for his suggestions, and Victoria Lebed for several useful comments and corrections. We also thank the referees for their careful review of our manuscript and valuable suggestions.

\section{REFERENCES}

[1] D. Bachiller. Classification of braces of order $p^{3}$. J. Pure Appl. Algebra, 219(8):3568-3603, 2015.

[2] D. Bachiller. Counterexample to a conjecture about braces. J. Algebra, 453:160-176, 2016.

[3] D. Bachiller, F. Cedo, and E. Jespers. Solutions of the Yang-Baxter equation associated with a left brace. arXiv:1503.02814.

[4] R. J. Baxter. Partition function of the eight-vertex lattice model. Ann. Physics, 70:193-228, 1972.

[5] R. J. Baxter. Exactly solved models in statistical mechanics. Academic Press, Inc. [Harcourt Brace Jovanovich, Publishers], London, 1989. Reprint of the 1982 original.

[6] N. Ben David and Y. Ginosar. On groups of central type, non-degenerate and bijective cohomology classes. Israel J. Math., 172:317-335, 2009.

[7] H. U. Besche, B. Eick, and E. A. O'Brien. A millennium project: constructing small groups. Internat. J. Algebra Comput., 12(5):623-644, 2002.

[8] W. Bosma, J. Cannon, and C. Playoust. The Magma algebra system. I. The user language. J. Symbolic Comput., 24(3-4):235-265, 1997. Computational algebra and number theory (London, 1993). 
[9] F. Catino and R. Rizzo. Regular subgroups of the affine group and radical circle algebras. Bull. Aust. Math. Soc., 79(1):103-107, 2009.

[10] F. Cedó, E. Jespers, and Á. del Río. Involutive Yang-Baxter groups. Trans. Amer. Math. Soc., 362(5):2541-2558, 2010.

[11] F. Cedó, E. Jespers, and J. Okniński. Retractability of set theoretic solutions of the YangBaxter equation. Adv. Math., 224(6):2472-2484, 2010.

[12] F. Cedó, E. Jespers, and J. Okniński. Braces and the Yang-Baxter equation. Comm. Math. Phys., 327(1):101-116, 2014.

[13] P. Dehornoy. Set-theoretic solutions of the Yang-Baxter equation, RC-calculus, and Garside germs. Adv. Math., 282:93-127, 2015.

[14] V. G. Drinfel'd. On some unsolved problems in quantum group theory. In Quantum groups (Leningrad, 1990), volume 1510 of Lecture Notes in Math., pages 1-8. Springer, Berlin, 1992.

[15] P. Etingof, T. Schedler, and A. Soloviev. Set-theoretical solutions to the quantum YangBaxter equation. Duke Math. J., 100(2):169-209, 1999.

[16] The GAP Group. GAP - Groups, Algorithms, and Programming, Version 4.7.8, 2015.

[17] T. Gateva-Ivanova. Set-theoretic solutions of the Yang-Baxter equation, Braces, and Symmetric groups. arXiv:150\%.02602.

[18] T. Gateva-Ivanova. A combinatorial approach to the set-theoretic solutions of the YangBaxter equation. J. Math. Phys., 45(10):3828-3858, 2004.

[19] T. Gateva-Ivanova. Quadratic algebras, Yang-Baxter equation, and Artin-Schelter regularity. Adv. Math., 230(4-6):2152-2175, 2012.

[20] T. Gateva-Ivanova and P. Cameron. Multipermutation solutions of the Yang-Baxter equation. Comm. Math. Phys., 309(3):583-621, 2012.

[21] T. Gateva-Ivanova and S. Majid. Set-theoretic solutions of the Yang-Baxter equation, graphs and computations. J. Symbolic Comput., 42(11-12):1079-1112, 2007.

[22] T. Gateva-Ivanova and S. Majid. Matched pairs approach to set theoretic solutions of the Yang-Baxter equation. J. Algebra, 319(4):1462-1529, 2008.

[23] T. Gateva-Ivanova and S. Majid. Quantum spaces associated to multipermutation solutions of level two. Algebr. Represent. Theory, 14(2):341-376, 2011.

[24] T. Gateva-Ivanova and M. Van den Bergh. Semigroups of I-type. J. Algebra, 206(1):97-112, 1998.

[25] E. Jespers and J. Okniński. Monoids and groups of I-type. Algebr. Represent. Theory, $8(5): 709-729,2005$.

[26] E. Jespers and J. Okniński. Noetherian semigroup algebras, volume 7 of Algebras and Applications. Springer, Dordrecht, 2007.

[27] C. Kassel. Quantum groups, volume 155 of Graduate Texts in Mathematics. Springer-Verlag, New York, 1995.

[28] V. Lebed and L. Vendramin. Homology of left non-degenerate set-theoretic solutions to the yang-baxter equation. arXiv:1509.07067.

[29] J.-H. Lu, M. Yan, and Y.-C. Zhu. On the set-theoretical Yang-Baxter equation. Duke Math. J., 104(1):1-18, 2000.

[30] S. Majid. Foundations of quantum group theory. Cambridge University Press, Cambridge, 1995.

[31] S. Nelson. The combinatorial revolution in knot theory. Notices Amer. Math. Soc., 58(11):1553-1561, 2011.

[32] W. Rump. A decomposition theorem for square-free unitary solutions of the quantum YangBaxter equation. Adv. Math., 193(1):40-55, 2005.

[33] W. Rump. Modules over braces. Algebra Discrete Math., (2):127-137, 2006.

[34] W. Rump. Braces, radical rings, and the quantum Yang-Baxter equation. J. Algebra, 307(1):153-170, 2007.

[35] W. Rump. Classification of cyclic braces. J. Pure Appl. Algebra, 209(3):671-685, 2007.

[36] W. Rump. The brace of a classical group. Note Mat., 34(1):115-144, 2014.

[37] A. Smoktunowicz. A note on set-theoretic solutions of the Yang-Baxter equation. arXiv:1512.95542.

[38] A. Soloviev. Non-unitary set-theoretical solutions to the quantum Yang-Baxter equation. Math. Res. Lett., 7(5-6):577-596, 2000. 
[39] M. Takeuchi. Survey on matched pairs of groups - an elementary approach to the ESS-LYZ theory. In Noncommutative geometry and quantum groups (Warsaw, 2001), volume 61 of Banach Center Publ., pages 305-331. Polish Acad. Sci., Warsaw, 2003.

[40] L. Vendramin. Extensions of set-theoretic solutions of the Yang-Baxter equation and a conjecture of Gateva-Ivanova. J. Pure Appl. Algebra, 220(5):2064-2076, 2016.

[41] A. Weinstein and P. Xu. Classical solutions of the quantum Yang-Baxter equation. Comm. Math. Phys., 148(2):309-343, 1992.

[42] C. N. Yang. Some exact results for the many-body problem in one dimension with repulsive delta-function interaction. Phys. Rev. Lett., 19:1312-1315, 1967.

Departamento de Matemática - FCen, Universidad de Buenos Aires, Pab. I - Ciudad Universitaria (1428) Buenos Aires - Argentina

E-mail address: leandroguarnieri@gmail.com

E-mail address: lvendramin@dm.uba.ar 